\title{
Magnetic fields in Herbig Ae stars
}

\author{
R. V. Yudin ${ }^{1}$, M. A. Pogodin ${ }^{1}$, S. Hubrig ${ }^{2}$ \\ and M. Schöller ${ }^{2}$ \\ ${ }^{1}$ Central Astronomical Observatory of the Russian Academy of Sciences at Pulkovo, 196140 \\ Saint-Petersburg, Russia, \\ email: ruslan61@gao.spb.ru; pogodin@gao.spb.ru \\ ${ }^{2}$ European Southern Observatory, Casilla 19001, Santiago 19, Chile, \\ email: shubrig@eso.org
}

\begin{abstract}
We present and discuss the results of a comprehensive analysis of magnetic field measurements for a group of seven young Herbig Ae/Be stars obtained using low-resolution spectropolarimetry with FORS1 at the VLT. The first definite evidence for the presence of surface magnetic fields has been found only recently in the Herbig Ae stars HD 139614, HD 31648 and HD 144432 (Hubrig et al. 2004; Hubrig et al. 2006) from the measurement of circular polarization in Ca II lines and hydrogen lines located in the spectral region from 3900 to $4900 \AA$. The unusual Herbig Ae star HD 190073 shows distinctive Zeeman features in the Ca II doublet which displays several components in both $\mathrm{H}$ and $\mathrm{K}$ lines. The most recent analysis carried out separately for different lines has shown that Zeeman features appear in metallic lines in all seven objects of our programme. The most prominent of them are seen in lines of the CaII doublet possibly of both photospheric and circumstellar origin. The circumstellar Balmer line components demonstrate the existence of a magnetic field in HD 144432 and HD 31648. The photospheric components of these lines with signs of magnetic field are present in HD 144668.
\end{abstract}

Keywords. Stars: pre-main-sequence, stars: magnetic fields, polarization, convection

\section{Introduction}

Numerous theoretical works predict the existence of a global magnetic field of a complex configuration around Herbig Ae/Be stars (HAEBEs). Nevertheless over an extended period of years all attempts to obtain reliable direct measurements of magnetic fields of HAEBEs have been rather unsuccessful. The lack of field detections could be explained by: (a) the weakness of the fields; (b) the low accuracy of all previous measurements; (c) possible time variability; and (d) non-adequate observational strategy. Quite recently, definite evidence for the presence of magnetic fields of the order of about 100G has been presented for several Herbig Ae stars by Hubrig et al. (2004); Hubrig et al. (2006). The accuracy of the field determinations was about $30 \mathrm{G}$ that allowed us to detect the magnetic fields in three HAEBEs at the $3 \sigma$ level. Those measurements have been carried out exclusively in the broad hydrogen lines. In the new analysis we have used the full spectrum, excluding only the regions which are contaminated by the circumstellar (CS) environment. The goals of our study are as follows: (a) to analyse the data of seven programme stars and to separate photospheric and CS components of all spectral lines; (b) to re-measure the photospheric magnetic fields of HAEBEs without inclusion of spectral regions affected by the CS matter; (c) to derive information on the presence of magnetic fields in the CS environment. 
Table 1. Results of magnetic field investigations

\begin{tabular}{lcccccc}
\hline Group & HD & MJD & $B_{z}$ previous & $B_{z}$ new & Stellar magnetic field & CS magnetic field \\
\hline I & 139614 & 53405 & $-116 \pm 34$ & $-93 \pm 14$ & $+(7 \sigma)$ & + \\
& 144432 & 53447 & $-119 \pm 38$ & $-111 \pm 16$ & $+(7 \sigma)$ & + \\
II & 38238 & 53249 & $-115 \pm 67$ & $+13 \pm 36$ & & + \\
& 139614 & 52904 & $-450 \pm 93$ & $-112 \pm 36$ & $?(3 \sigma)$ & + \\
& 144668 & 52901 & $-118 \pm 48$ & $+166 \pm 40$ & $?(4 \sigma)$ & + \\
III & 144668 & 53120 & $-107 \pm 40$ & $-75 \pm 29$ & & + \\
& 31648 & 53296 & $+87 \pm 22$ & $+73 \pm 32$ & & + \\
& 163296 & 53279 & $-57 \pm 33$ & $-25 \pm 27$ & & + \\
& 190073 & 53514 & & $+21 \pm 12$ & & + \\
& 190073 & 53519 & $+84 \pm 30$ & $+14 \pm 22$ & & $+(3 \sigma)$ \\
\hline
\end{tabular}

\section{Observations}

For the measurement of the magnetic fields we used spectropolarimetric observations obtained with the $8 \mathrm{~m}$ VLT+FORS1 in the years 2003-2005. A detailed description is presented in our previous papers (Hubrig et al. 2004; Hubrig et al. 2006).

\section{Results and discussion}

The results of our analysis are summarized in the table 1. We suggest that the observations of the studied HAEBEs can be divided into three groups. The first group contains objects with photospheric magnetic fields which have been detected at a high confidence level of about $7 \sigma$. The objects with only weak circular polarisation signatures which however cannot be determined with sufficient precision constitute the second group. Objects with clear circular polarisation signatures mainly of CS origin have been distinguished as a third group. The differences are illustrated in the figures below.

\section{Conclusions}

- We conclude that previous discrepancies in estimations of magnetic fields for a few HAEBEs observed on different dates are likely the result of a variable CS contribution to photospheric spectra.

- We have improved the accuracy of the photospheric magnetic field determination of two objects (HD139614, HD144432) to a significance level of about $7 \sigma$. This leaves little doubt about the presence of a magnetic field.

- We deduce that the measured magnetic fields of HD31648 and HD190073 are not of photospheric but mostly (HD31648) or exclusively (HD190073) of CS origin.

- We found that the most sensitive indicator of the CS magnetic field in Herbig Ae stars is the CaII doublet. Circular polarisation features corresponding to this doublet are observed in all programme targets of groups II and III. The magnetic field diagnosed in the CaII lines is generated in the CS matter in the vicinity of the stellar surface where the base of the stellar wind as well as gaseous flows infalling onto the star are likely located.

- From the analysis of polarisation signatures in HD 31648 and HD 144432 we conclude that in the Balmer lines they are only observed in blueshifted absorption components formed in a dense stellar wind. All the above can be considered in favor to the presence of significant convection zones in stellar envelopes of Herbig Ae stars (cf. Giardino et al. (2004); Strafella et al. (1998)). 

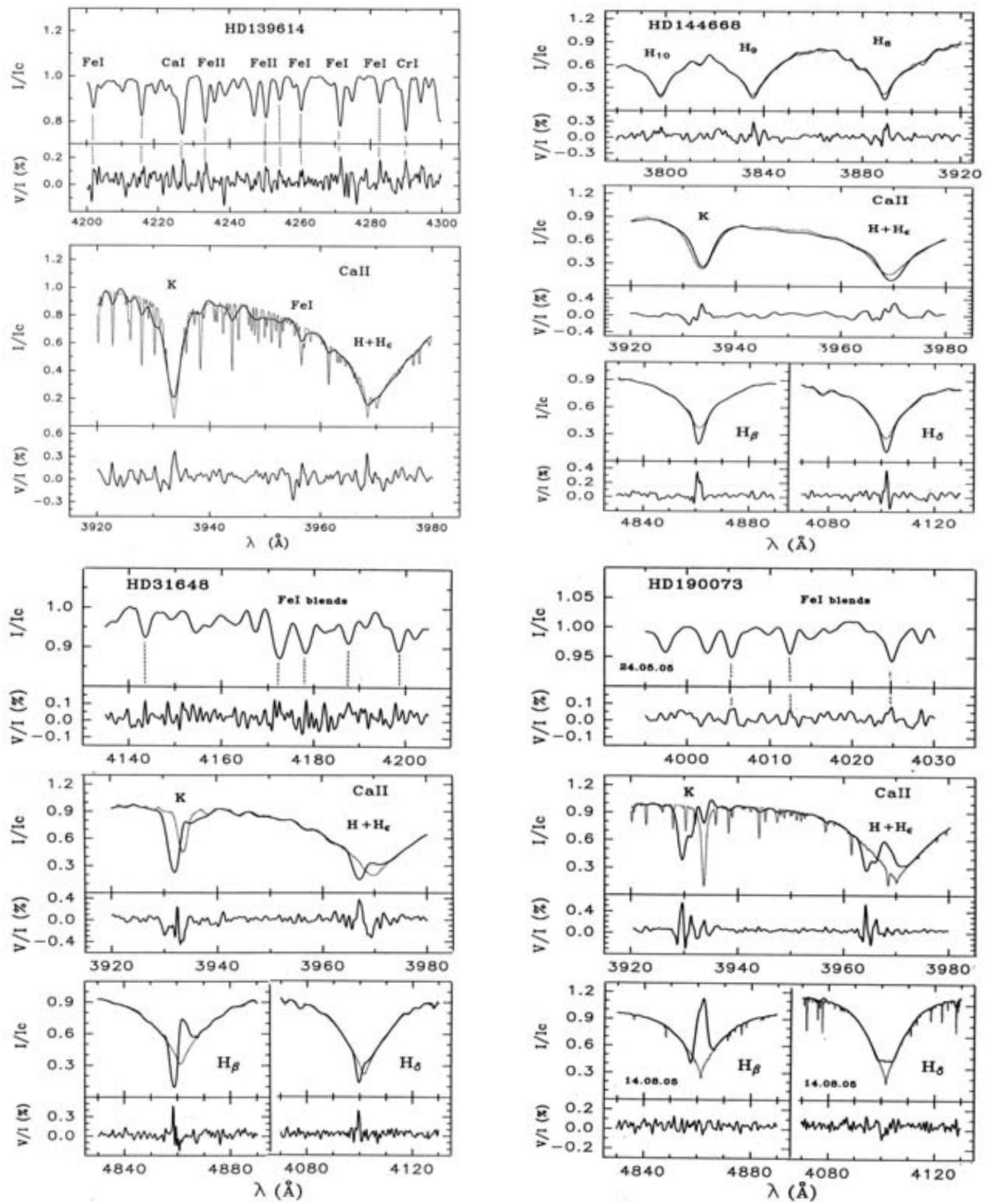

Figure 1. Stokes I and V spectra in different spectral regions. The dotted vertical lines indicate polarisation signatures corresponding to positions of stellar atmospheric lines. The synthetic photospheric spectrum is shown in the other panels by the dotted line.

\section{References}

Giardino, G., Favata, F., Micela, G. \& Reale, F. 2004, A\&\&A 413, 669

Hubrig, S., Schöller, M. \& Yudin, R. V. 2004, A\& A 428, L1

Hubrig, S., Yudin, R. V., Schöller, M. \& Pogodin, M.A. 2006, A\& A 446, 1089

Strafella, F., Pezzuto, S., Corciulo, G.G., et al. 1998, ApJ 505, 299 